\title{
The Comparison and research in low power consumption between ZigBee and Bluetooth technologies
}

\author{
Fang Dong \\ (Anhui university Anhui Hefei 230601)
}

\begin{abstract}
Keywords: ZigBee technology; Bluetooth technology; Low power consumption; Short distance wireless communication
\end{abstract}

\begin{abstract}
ZigBee and Bluetooth are the wireless communication technologies that play an important role in wireless ad-hoc networks. This paper analyzes and compares different low-power control strategies used by two kinds of technique standards, furthermore, we observe the current consumption, and then come to a reasonable conclusion through the tests of two kinds of typical applying circuit. ZigBee has a significant advantage on low-power consumption. While the Bluetooth 4.0 combines high-speed and low-power consumption, and to a large extent, it reduces the power consumption. In general, ZigBee technology and Bluetooth technology have their own advantages, as well as they complement for and compete with each other.
\end{abstract}

\section{A INTRODUCTION}

ZigBee and Bluetooth are both IEEE802.15 series, the WPAN (Wireless Personal Area Networks) wireless communication standard specification.The main features of this series of standards is a short-range, low-power, low-cost, small-scale networking and communications equipment for personal operating space.ZigBee is a low rate wireless communication technology defined by the working group IEE802.15.4 to fit for cheap, low complexity portable or mobile devices,mainly suitable for automatic control, sensing, monitoring and remote control, etc. Bluetooth is defined by the IEEE802.15.1 for supporting a short-range radio technology communication devices,mainly used between the mobile phone, PDA, wireless headsets, laptops and other personal devices.In terms of low-power Bluetooth deficiency,Bluetooth SIG has also filed a Bluetooth version 4.0,namely,Bluetooth low energy.

Now with the large-scale popularity of mobile Internet, ZigBee and Bluetooth technology is increasingly widely used in all aspects of life,the low power consumption has become a major consideration.In many application scenarios, it needs to consider the the lowest power consumption,so that equipment can run much longer.This paper analyzes the characteristics of ZigBee and Bluetooth from the view of international standards in low-power control strategy,then select the typical unit circuit to test. This paper has a good practical significance for the selection of two applications of wireless communication technologies.

\section{B INTERNATIONAL STANDARDS IN LOW POWER STRATEGY BETWEEN ZIGBEE AND BLUETOOTH}

\section{B.A LOW POWER STRATEGY IN ZIGBEE}

ZigBee technology includes the Media Access Control layer (MAC) and Physical Layer(PHY), at the beginning of its standards, it includes a variety of low-power control mechanisms. 


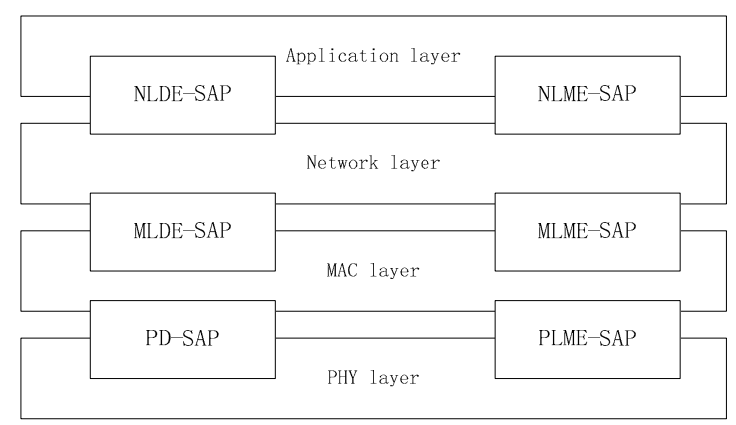

Fig 1.ZigBee protocol stack structure

(1)The terms of the protocol stack

MAC layer protocol is designed to reduce power consumption, which main role is to encapsulate frames, to extract physical information and analytic frame if it is true or false,.MAC layer is consisted with frame header, data, and end of frame.To further reduce power consumption, IEEE 802.15.4 working group improves the MAC layer, there are S-MAC (Self-organizing MAC) layer and T-MAC (Timeout-MAC) layer. S-MAC listens sleep mode at a low duty cycle periodically, and let more time in sleep mode to reduce unnecessary delays by decreasing adaptive listening. T-MAC is on the basis of S-MAC, you can dynamically change the length of time depending on traffic, which can further reduce the active time and power consumption.

(2)Topological structure

ZigBee mainly has star, tree and mesh types . These types have their own characteristics,.In terms of networking, because of the large nodes and network scale, the use of different technologies for power consumption also has a great impact. Star structure has a central controller and a number of sub-device, which requires the central controller must be a full-function device, therefore it will cost greater energy consumption. Tree types is widely used, it is divided into the trunk and branches, trunk part equipment is fully functional device, only in the distal branches that can be reduced function devices,.Compared to the star structure, the tree structure has more resources and it is more stable and reliable. Mesh-type structure is more complex and the most flexible, every device can communicate with every peripheral equipment, which makes the mesh-type structure has the greatest power consumption compared to the star and tree topology . Therefore, from the low power of view, we should try to avoid using the network topology.

(3) link-state mode

ZigBee has active and dormant mode,.In the sleep state, the host will be in the ultra-low-load condition, only when it is needed by the controller to start the operation, the RF energy is almost negligible. we can wake it up by timer or external buttons, it can reduce the power consumption to a large extent .

\section{B.B LOW POWER STRATEGY IN BLUETOOTH}

Bluetooth technology is based on the IEEE802.15.1 standard, and now has developed to the 4.0 version, this version is the combination of traditional Bluetooth (Bluetooth $2.1+$ EDR), high-speed Bluetooth (Bluetooth 3.0 + HS) and low-power Bluetooth (Bluetooth 4.0) integration. It can be used alone and in combination. In the Bluetooth 4.0 standard, Bluetooth low energy technology BLE (Bluetooth Low Energy) has been widespread concerned. BLE can reduce the power consumption to a large degree compared to traditional Bluetooth.

\begin{tabular}{|c|c|}
\hline Generic Access Profile(GAP) & $\begin{array}{c}\text { Genetic Attribute } \\
\text { Profile(GATT) }\end{array}$ \\
\hline Security Manger(SM) & Attribute Protocol(ATT) \\
\hline Logical Link Control and Adaption Protocol (L2CAP) \\
\hline Host Controller Interface(HCI) \\
\hline Link Layer(LL) \\
\hline Physical Layer(PHY) \\
\hline
\end{tabular}

Fig 2.Bluetooth 4.0 protocol architecture 
Bluetooth low energy protocol can be divided into control part, host part and application part. We can see from the Fig 2, the control section includes a physical layer (PHY) and a link layer (LL layer), the host portion is comprised by a logical link control and adaptation protocol (L2CAP), security management (SM) and attribute protocol (ATT), application part is the corresponding APP.

The low energy is mainly reflected in the following aspects:

(1)The terms of the protocol stack

Classic Bluetooth has a variety of protocols, there are many different types of specifications and data packets, it is redundant and complex and leading to high energy consumption. The design of 4.0 version is simplified, it only uses a attribute on the structure thereby reducing power consumption more efficient.

(2)The terms of connection events

Bluetooth 4.0 protocol for the connection event set three parameters: connection interval, the delay and the time-out from the device. Connection interval in units of $1.25 \mathrm{~ms}$, the minimum can be set to $7.5 \mathrm{~ms}$, the maximum can be set to $4.0 \mathrm{~s}$; refers to the delay from the device in the absence of data to be transmitted, from the device may skip a number of connection event without replying to the main equipment,so can decrease the power consumption; we set a maximum time for time-out form when the connection time is longer than the maximum it will be automatically disconnected.

(3)link-state patterns

Bluetooth has four link-state model. It is active mode, breathing mode, keep mode and dormancy mode. It has higher power consumption in active mode, the master device sends data periodically, The hold mode is setting a certain holding time, at this time, so the bluetooth module can go to sleep, it will wake up more than this predetermined time. Breathing mode is a figurative description, as human breathing, there is a certain gap between the two breathing, and only in this gap can listen for the data packet,when not listening it cab be back to sleeping mode.

\section{TYPICAL CIRCUIT TEST IN LOW POWER COMPARISON}

Using the indirect method,Scheme shown in Figure 3,Choosing a typical ZigBee, Bluetooth radio channel module respectively, as measured target board, tested under different working conditions of the power situation.

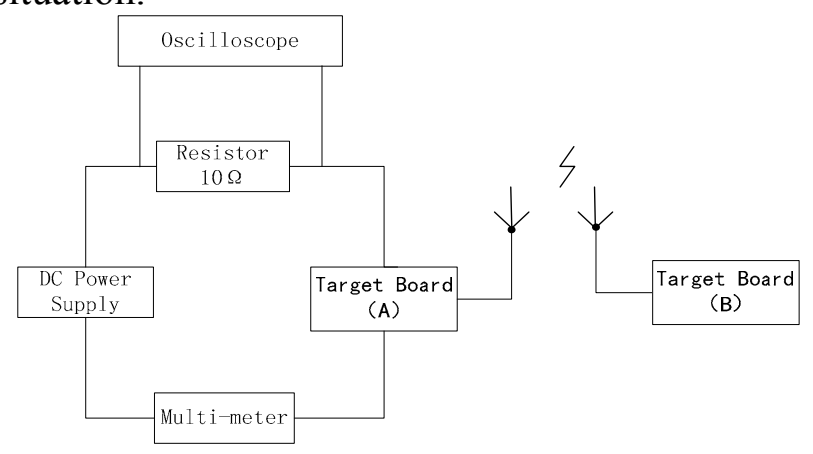

Fig 3.Measurement Setup

Where $\mathrm{V}$ is the voltage, $\mathrm{R}$ is the resistance and $\mathrm{I}$ is the current. By measuring at the power supply side, the test system will observe the current consumed by the complete target board.

Noting that $\mathrm{R}$ should not be too large, since it will reduce the effective voltage over the target

board itself.

$$
V_{t \text { arget }}=V_{D C}-(I \times R)
$$

However, the value also can not be made too small, which makes the voltage across the resistor is too small, it will result in insufficient accuracy of the oscilloscope to enhance error.

Once the current is determined, the overall average current consumption can be found using the general formula

$$
I_{\text {avg }}=\sum_{i=0}^{n}\left(\frac{T_{i}}{P_{i}} \times I_{i}\right)+\left(1-\sum_{i=0}^{n}\left(\frac{T_{i}}{P_{i}}\right)\right) \times I_{\text {sleep }}
$$


$T_{i}$ is equipment of average current consumption time, $P_{i}$ is average consumption test of the time; $I_{\text {sleep }}$ is the sleep mode current consumption, $I_{\text {avg }}$ is the total time of testing the average consumption current.

Finally can calculate that the life time of the system:

Lifetime $(\mathrm{h})=\frac{\text { Battery capacity }(\mathrm{mAh})}{\text { Average current }(\mathrm{mA})}$

\section{C.A ZIGBEE LOW POWER SYSTEM}

Using a pair of single-chip CC2530 minimum system modules respectively, as the transmitter (target A) and receiver (target B)Using oscilloscope to test the system, the results shown in Figure 4, one of the data during transmission. Then you can also use a digital multi-meter measured emission current..

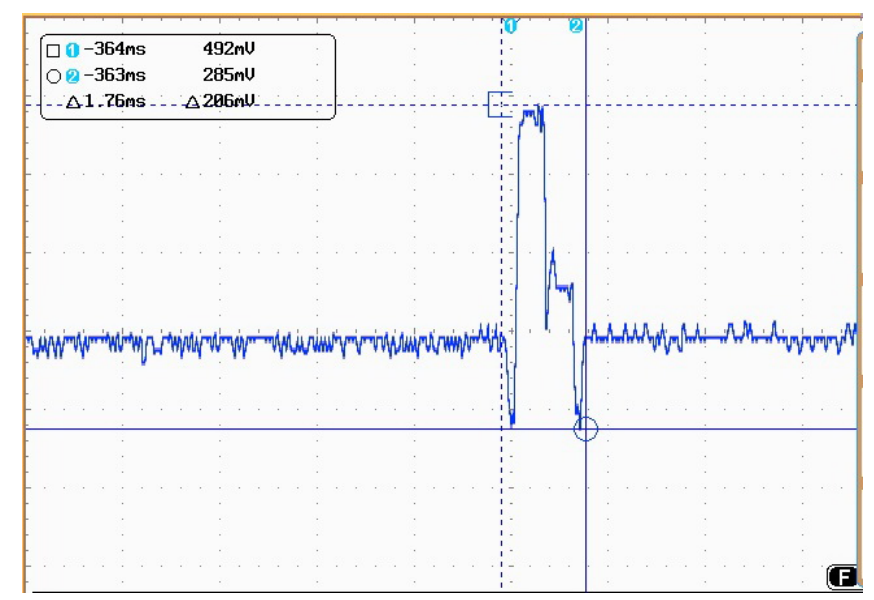

Fig 4.ZigBee transmitter oscilloscope test image

The wave represents that CC2530 transmitter is sending the controlling signal, the data packets is transmitted by wireless. After the end of the transmissionCC2530will return to sleep.

C.B BLUETOOTH LOW POWER SYSTEM

For ease of comparison, we use the same test program for testing the Bluetooth module, a pair of minimum system CC2540 Bluetooth modules respectively, regarded as measured target A and B.

There are four types of low-power Bluetooth devices: Central Host, Peripheral slave, Observer observer and Broadcaster broadcasters,.In this test there will be a Bluetooth device A as the host, the other Bluetooth device B as a slave, press button on A and control LED lights on B .

The difference is that the Bluetooth should search sub-devices first.Communicating with each other based on low-power Bluetooth GATT (Generic Attribute Profile) layer, there are only two Bluetooth module can communicate with each other between the same specification, through profile we can define this specification, when the host is connected to its corresponding slave then the slave can communicate data.

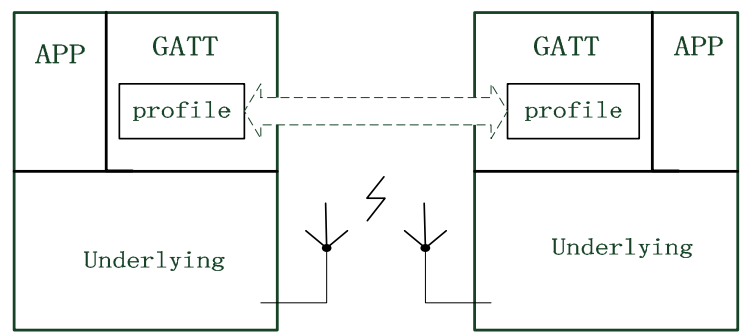

Fig 5.Bluetooth master and slave data transmission

After power serial commands via the host device search, in this process will continue to send the host module search signal, power consumption is greatly improved. If no device is found from the machine into standby, after searching can be controlled from the machine. Fluctuations in the figure represents CC2540 Bluetooth device receives serial commands are searching for. 


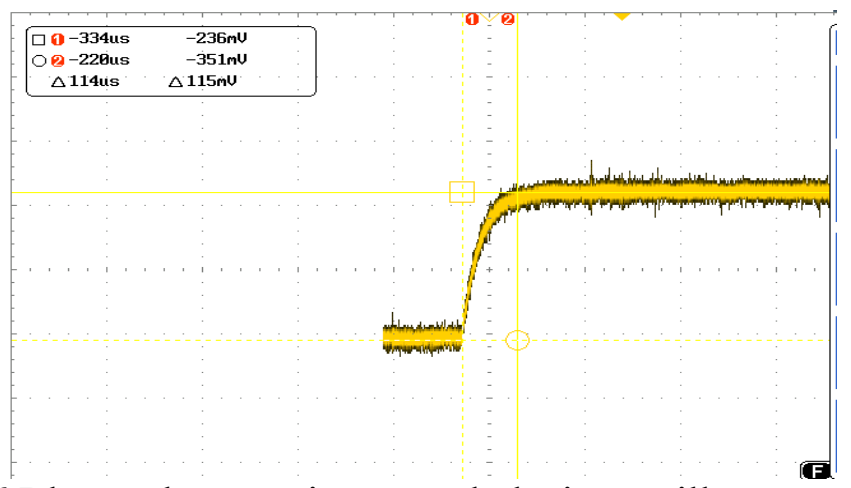

Fig 6.Bluetooth transmitter search device oscilloscope test image

\section{C.C LOW POWER ANALYSIS}

Using the same test above the scheme, compared the results between ZigBee and Bluetooth 4.0, the data shown in Table 1.

Table 1. The results

\begin{tabular}{ccc}
\hline performance & ZigBee & Bluetooth4.0 \\
\hline Test module & CC2530 & CC2540 \\
The way of spread & DSSS & FHSS \\
Serial interface & $3.3 \mathrm{~V} \mathrm{TTL}$ & $3.3 \mathrm{~V} \mathrm{TTL}$ \\
A serial port baud rate & $115.2 \mathrm{Kbps}$ & $115.2 \mathrm{Kbps}$ \\
Bottom currents & $19.0 \mathrm{~mA}$ & $19.0 \mathrm{~mA}$ \\
Waking up the current & $20.2 \mathrm{~mA}$ & $24.1 \mathrm{~mA}$ \\
& & Search $33.7 \mathrm{~mA}$ \\
Emission current & $34.0 \mathrm{~mA}$ & Transmitting \\
(Only one sub-device) & & $24.4 \mathrm{~mA}$ \\
Frequency range & $2402-2480 \mathrm{Mh}$ & $2402-2480 \mathrm{Mhz}$ \\
The core plate voltage & $3-3.5 \mathrm{~V}$ & $3-3.5 \mathrm{~V}$ \\
Link-state mode & Active/sleep & Active/breathing/ \\
Power consumption & low & low \\
\hline
\end{tabular}

By testing,we can find it that ZigBee has a smaller power consumption in the wake mode and after waking up it can transmit signals as soon as possible. But Bluetooth can not send data directly when wake it up.According to thr GATT (Generic Attribute Profile) layer it must establish the same specification between two Bluetooth devices that can transfer data, so the first step is to search for the matched device and set a connection, the emission current in this part is similar to ZigBee's, and is the major power consuming in Blutooth. As long as Bluetooth devices working in this condition, you can continue to send data without searching again, at this time,the transmitter current is much smaller than the emission current ZigBee emission current. At the same time we test the operating current of dormancy in the two modules, both in the microampere level, indicating that the current sleep mode is very small, so it can be negligible.

\section{CONCLUSION}

By comparison, ZigBee and Bluetooth 4.0 have a low power consumption, but their protocols are different. ZigBee commonly used in commercial and residential devices otherwise Bluetooth is often used in personal device due to its limitation in its volume and distance o But no matter what kind of technology, there is not only technology itself, but other factors, such as the market impact 
also has great influence. It is foreseeable that the Bluetooth SIG and ZigBee technology alliances will be more intense with each other in the future. When selecting communications technology for different products, it should depend on the actual situation such as network size, communication range, power consumption, security, etc., including a variety of factors to weigh the advantages and disadvantages, and then to determine one technologies and products. From this perspective, in order to be able to provide better solutions to meet such needs, Zigbee and Bluetooth also need become more dependent on each other partnerships.

\section{REFERENCES}

[1]Suyash Jain, Morten Braathen. Measuring the Power Consumption on CC2530ZNP Using CC2530 ZNP Mini Kit[Z]. TEXAS: TEXAS INSTRUMENTS INCORPORATED,2011.

[2]RF-PHY.TS.4.0.0,Bluetooth Low Energy RF PHY Test Specification.

[3]LPRF San Diego Bluetooth Low Energy Deep Dive[Z]. TEXAS:TEXA INSTRUMENTS INCORPORATED, 2011.

[4]Bluetooth SIG.Specification of the Bluetooth System: Core.vol. 1.1, 2001

[5]IEEE802.15.4: Wireless Medium Access Control (MAC)and Physical Layer (PHY) Specifications for Low Rate Wireless Personal Area Networks(LR-WPANs) , October, 2003 